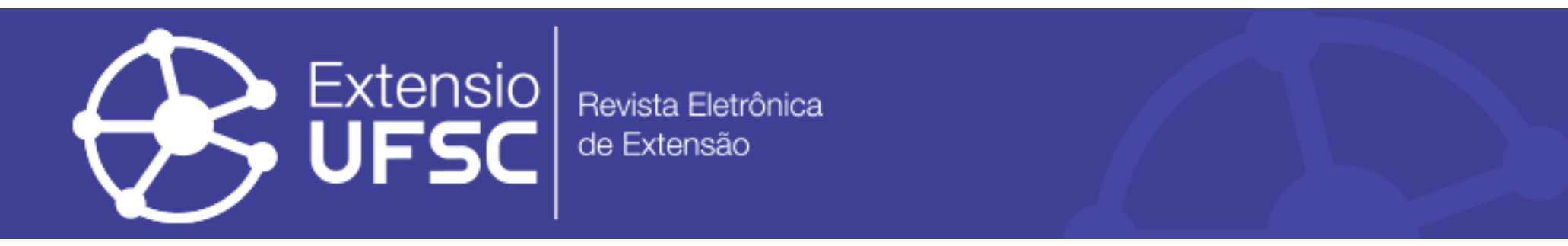

\title{
A PARTICIPAÇÃO DA EXTENSÃO NA RESOLUÇÃO DE CASO DE CRIANÇA COM DIABETES MELLITUS TIPO 1
}

\author{
Marcia de Freitas Oliveira \\ Universidade Regional de Blumenau \\ marciaoliveira@furb.br \\ Nevoni Goretti Damo \\ Universidade Regional de Blumenau \\ nevoni@furb.br
}

\author{
Caroline Lopes \\ Universidade Regional de Blumenau \\ dra.carolopes@gmail.com \\ Isadora Wileman Raitz \\ Universidade Regional de Blumenau \\ isadoraraitz@hotmail.com \\ Liandra Pereira \\ Universidade Regional de Blumenau \\ liandrapereira0605@gmail.com
}

\section{Resumo}

Introdução: Diabetes Mellitus (DM) representa um grupo de doenças caracterizadas por hiperglicemia. Objetivo: foi demonstrar a importância da extensão, da atuação interprossional e multiprofissional para a resolução de um caso clínico dentro de um Projeto de extensão interprofissional na perspectiva de clínica ampliada. Metodologia: foi realizado relato da atuação da extensão universitária em um caso de uma criança de 11 anos de idade com Diabetes mellitus tipo 1 (DMT1) não controlada, apresentando um quadro de hiperplasia gengival, além de fratura incisal no dente 21. Resultados: O protocolo de atendimento foi preventivo e terapêutico e incluiu: aferição da glicemia capilar, antibioticoterapia profilática, atendimento interprofissional e multiprofissional e controle do quadro de hipoglicemia. $\mathrm{O}$ apoio dos familiares foi fundamental para o sucesso do tratamento. Conclusão: Conclui-se que a atuação interprofissional e multiprofissional no planejamento e no desenvolvimento das atividades, favoreceram a interlocução de conhecimentos e experiências para ajudar na resolução das situações vivenciadas, promovendo, assim, melhores condições para o paciente lidar com sua saúde geral e bucal.

Palavras-chave: Diabetes Mellitus. Hipoglicemia. Manifestações Bucais.

\section{THE PARTICIPATION OF EXTENSION IN THE RESOLUTION OF CHILDREN'S CASE WITH DIABETES MELLITUS TYPE 1}

Abstract

Introduction: Diabetes Mellitus (DM) represents a group of diseases characterized by hyperglycemia. Objective: it was to demonstrate the importance of extension, interprossional and multiprofessional action for the resolution of a clinical case within an Interprofessional Extension Project in the perspective of expanded clinic. Methodology: a report was made of the performance of university extension in a case of an 11-year-old child with uncontrolled type 1 Diabetes mellitus (DMT1), presenting a picture of gingival hyperplasia, in addition to incisal fracture in tooth 21. Results: O The care protocol was preventive and therapeutic and included: measurement of capillary blood glucose, prophylactic antibiotic therapy, interprofessional and multiprofessional care and control of hypoglycemia. The support of family members was essential for the success of the treatment. Conclusion: It is concluded that the interprofessional and multiprofessional performance in the planning and development of activities, favored the exchange of knowledge and experiences to help in solving the situations experienced, thus promoting better conditions for the patient to deal with his general and oral health. Keywords: Diabetes Mellitus. Hypoglycemia. Oral Manifestations.

\section{LA PARTICIPACIÓN DE LA EXTENSIÓN EN LA RESOLUCIÓN DEL CASO INFANTIL CON DIABETES MELLITUS TIPO 1}

\section{Resumen}

Introducción: La diabetes mellitus (DM) representa un grupo de enfermedades caracterizadas por hiperglucemia. Objetivo: fue demostrar la importancia de la extensión, la acción interprossional y multiprofesional para la resolución de un caso clínico dentro de un Proyecto de Extensión Interprofesional en la perspectiva de la clínica ampliada. Metodología: se realizó un informe sobre el desempeño de la extensión universitaria en un caso de un niño de 11 años con diabetes mellitus tipo 1 no controlada (DMT1), presentando una imagen de hiperplasia gingival, además de fractura incisal en el diente 21 . Resultados: $\mathrm{O}$ El protocolo de atención fue preventivo y terapéutico e incluyó: medición de la glucosa en sangre capilar, terapia antibiótica profiláctica, atención interprofesional y multiprofesional y control de la hipoglucemia. El apoyo de los miembros de la familia fue esencial para el éxito del tratamiento. Conclusión: Se concluye que el desempeño interprofesional y multiprofesional en la planificación y desarrollo de actividades, favoreció el intercambio de conocimientos y experiencias para ayudar a resolver las situaciones experimentadas, promoviendo así mejores condiciones para que el paciente se ocupe de su salud general y oral.

Palabras clave: Diabetes Mellitus. Hipoglucemia. Manifestaciones Orales. 


\section{INTRODUÇÃO}

O Diabetes Militus (DM) representa um grupo de doenças caracterizadas por hiperglicemia, como resultado da deficiência na produção de insulina, resistência periférica à sua ação, ou ambas (WHO, 1999). Em indivíduos saudáveis, a concentração plasmática de glicose situa-se entre 70 e $99 \mathrm{mg} / \mathrm{dL}$, em jejum. Níveis superiores indicam graus variados de tolerância à glicose (pré-diabetes) ou diabetes, cujos critérios de diagnóstico foram atualizados pela American Dental Association (ADA, 2019). As duas formas mais comuns de DM são: Diabetes Mellitus tipo 1 (DMT1) e Diabetes Mellitus tipo 2 (DMT2). Outras formas menos comuns são: o diabetes gestacional, diabetes associado a medicamentos (corticoides), doenças do pâncreas (fibrose cística), infecções (rubéola congênita) e síndromes genéticas (OLIVEIRA et al., 2019).

O DMT1 é uma doença autoimune, decorrente da destruição gradual das células betas pancreáticas (WITT et al., 2010; WHO, 1999; SBD, 2020), comumente de causa desconhecida. Esse tipo de diabetes possui dois picos de incidência, entre 5-7 anos e na puberdade, sem predileção por sexo. Essa patologia acomete 5 a 10\% dos pacientes com DM. Quando o quadro clínico se inicia, já houve perda de 80 a $90 \%$ da capacidade de produção de insulina. As manifestações clássicas do DMT1 incluem poliúria (aumento do volume urinário, devido à diurese osmótica, causada pelo excesso de glicose), polidipsia (aumento da sede para compensar a perda de água pela urina), polifagia (aumento da fome, para compensar o estado catabólico resultante da deficiência de insulina) e perda de peso, devido às células serem incapazes de captarem glicose (ANAD, 2020; SBD, 2020, ALVES et al., 2006; AGUIAR et al., 2009; SANTOS et al., 2010).

Já no DMT2 a destruição autoimune do pâncreas não está envolvida, e sim decorre da combinação de deficiência relativa de insulina e resistência insulínica, sendo a forma mais comum da doença, responsável por $90-95 \%$ dos casos. A idade de início é variável, embora seja mais frequente após os 40 anos. Geralmente associada à obesidade, hipertensão e dislipidemia. Um número significativo de pessoas com DMT2 é assintomática ou oligossintomática (SBD, 2020). O tratamento farmacológico indicado para DMT1 é, imprescindivelmente, a insulinoterapia, e para a DMT2 são indicados desde os hipoglicemiantes orais à insulinoterapia e, para ambos, modificações no estilo de vida (ANAD, 2020, SBD, 2020).

A incidência do DM vem atingindo proporções epidêmicas. Segundo relatório da Federação Internacional de Diabetes, este é um dos problemas de saúde que mais cresce, estimase que até 2030578 milhões e até 2045700 milhões de pessoas tenham diabetes. O relatório descreve que o Brasil possui o maior número de adultos com diabetes, 16,8 milhões, sendo a 
prevalência maior em mulheres do que em homens. Ainda descreve que o Brasil é o terceiro país do mundo em número de crianças e adolescentes com DMT1 (FEDERAÇÃO INTERNACIONAL DE DIABETES, 2019).

Entre as principais manifestações bucais dos pacientes com diabetes não controlados estão: gengivite, periodontite e infecções fúngicas bucais recorrentes (FERNANDES et al., 2010). Os tecidos periodontais são as estruturas mais afetadas pelo DM, sendo que a doença periodontal é considerada pela Organização Mundial da Saúde (OMS) como a sexta complicação crônica do distúrbio metabólico. Quanto mais cedo ocorre o aparecimento do diabetes e quanto maior o período de descompensação da doença, mais suscetível será o paciente com diabetes a desenvolver doença periodontal (TERRA et al., 2011). Lima et al. (2015), em uma revisão de literatura, evidenciaram que a doença periodontal encontra-se presente em $75 \%$ dos casos, sendo que um controle ruim do diabetes, a longo prazo, leva a lesões microvasculares do periodonto.

A periodontite pode alterar a condição sistêmica em pacientes com diabetes e, portanto, interferir no resultado do controle glicêmico (BOTERO et al., 2013), como também, o controle metabólico do diabetes provoca melhora nas circunstâncias periodontais (CIRIACO e BUENDÍA, 2014). A presença de infecções como a doença periodontal leva à super estimulação da resposta inflamatória, resultando em situação de estresse, que aumenta a resistência dos tecidos à insulina, piorando o controle do diabetes (TERRA et al., 2011). Portanto, é fundamental que todos os cirurgiões-dentistas adquiram conhecimento sobre o diabetes e sua influência na saúde periodontal para saber diagnosticar e tratar esses pacientes (OLIVEIRA et al., 2019).

Fernandes et al. (2015) em um estudo prospectivo caso-controle com 53 participantes com DMT2 e 29 participantes que não tinham DMT2, os quais foram submetidos extrações de dentes erupcionados, observaram que os pacientes com diabetes têm um atraso de epitelização, contudo o mesmo não se relaciona com infecção ou dificuldade de reparo e hiperglicemia Apesar de pessoas com DMT2 poderem ter função neutrofílica prejudicada, os resultados do estudo revelaram também que essa condição não estava associada com risco aumentado de sofrer complicações pós-operatórias. Os autores também sugerem que estudos de pesquisa adicionais, com amostras maiores de pacientes, são necessários para confirmar as descobertas deste estudo.

O tratamento odontológico mal orientado em paciente diabético descompensado implica grave risco à sua saúde sistêmica, devido às complicações agudas desta doença (BORGES et al., 2010). As principais complicações agudas do DM são: a hipoglicemia, caracterizada pela glicemia abaixo de $70 \mathrm{mg} / \mathrm{dL}$ e a hiperglicemia, caracterizada pela glicemia maior de $160 \mathrm{mg} / \mathrm{dL}$ (ALVES et al., 2006, SANTOS \& JUNIOR, 2009). A aferição da glicemia é realizada por meio do aparelho denominado glicosímetro. 
A rede de apoio social, as relações familiares e de confiança com os profissionais de saúde influenciam nos comportamentos de autocuidado e autocontrole, além de aumentar a adesão ao tratamento, resultando na melhora do controle glicêmico (NASCIMENTO et al., 2011; LOPES at al 2019). O envolvimento de uma equipe interprofissional para o auxílio às limitações físicas, alimentares e de socialização é importante para um manejo adequado. Torna-se, portanto, indispensável o acompanhamento multiprofissional e interprofissional, considerando a individualidade, conduzindo-os a uma vida saudável a partir de suas potencialidades.

O objetivo deste estudo foi demonstrar a importância da extensão, da atuação interprossional e multiprofissional para a resolução de um caso clínico dentro de um Projeto de extensão interprofissional na perspectiva de clínica ampliada.

\section{METODOLOGIA}

O projeto de extensão "Doce Sorriso e apoio ao autocuidado medicamentoso" é um projeto de extensão que tem como objetivo proporcionar atenção básica em saúde bucal a crianças/adolescentes com diabetes, a partir de uma visão interprofissional e multiprofissional, além de acompanhar e orientar as crianças com DMT1 quanto ao tratamento farmacológico e não farmacológico.

Paciente do sexo feminino, de onze anos de idade, compareceu à clínica odontológica da Universidade Regional de Blumenau (FURB), onde participou do projeto de extensão Doce Sorriso e apoio ao autocuidado medicamentoso, para avaliação odontológica e farmacêutica (interprofissional), o qual também promove a integração dos pacientes/familiares e acadêmicos de diversos cursos da área da saúde.

O projeto possui ações de promoção à saúde geral e bucal que envolvem estratégias de educação em saúde (murais, folder, vídeos, rodas de conversas, redes sociais) que enfatizam o conceito de saúde geral e bucal e os meios para mantê-las saudáveis, como a escovação e o cuidado com os medicamentos, entre outras, para pacientes, professores, pais e/ou cuidadores, incentivando-os quanto ao desenvolvimento de novos hábitos de higiene oral e a importância do cuidado para a saúde geral.

Diante disso, os acadêmicos que participam deste projeto têm a oportunidade de trabalhar em conjunto com as diversas áreas de formação em saúde. Esta integração permite a ampliação e a consolidação do conhecimento a respeito da área de escolha para atuação profissional, por meio de trocas de abordagens e experiências, atingindo as metas de um projeto de extensão (ANGIONI, 2015). 


\section{RELATO DE CASO E ANÁLISE}

No dia do primeiro contato com o projeto de extensão, a paciente compareceu com sua avó, que é a responsável pela criança, momento no qual foi realizada aferição da glicemia, constatando o valor de $410 \mathrm{mg} / \mathrm{dL}$. Além disso, foi realizado exame clínico, exame radiográfico e assinado o termo de consentimento para tratamento e uso de dados clínicos para publicação.

Durante a anamnese foi relatado que a paciente está sob cuidados médicos. Segundo relato familiar, o diabetes foi diagnosticado em 2009, aos oito anos de idade, quando a paciente necessitou internação na UTI devido à cetoacidose. Após esse episódio não houve mais internações. Faz uso de insulina NPH e regular. A avó tem dificuldade de monitorar a dieta da paciente, a qual não era balanceada e nem acompanhada por nutricionista. Foram relatados diversos quadros de hiperglicemia. Atualmente, reside com os avós e uma tia. O pai acompanha o desenvolvimento da criança e reside em uma cidade vizinha. Durante a história odontológica, a avó relatou que a paciente nunca havia sido submetida à anestesia odontológica, não faz uso de flúor, possui hábitos de bruxismo noturno e onicofagia. Os hábitos de higiene como escovação mecânica dental se iniciaram aos seis meses de idade. Atualmente a escovação mecânica é realizada pela criança três vezes ao dia, porém, com uso de fio dental em períodos ocasionais.

$\mathrm{Na}$ avaliação bucal pôde-se observar a presença de cálculo dental supragengival nos incisivos inferiores, nas faces vestibular e lingual, mesial e distal. Apresentava hiperplasia gengival e gengivite no rebordo alveolar ântero-inferior. Observou-se fratura no dente 21 envolvendo a incisal no terço mesial, devido a uma queda durante brincadeira. Não era respiradora bucal.

No segundo encontro com a criança no projeto de extensão, para tratamento odontológico realizou-se a glicemia capilar e constatou-se glicemia casual pré-consulta, de 460 $\mathrm{mg} / \mathrm{dL}$, porém nenhum sintoma foi relatado pela criança. Paciente compareceu acompanhada do pai, o qual mostrou interesse pelo tratamento e decidido a cooperar. Foram realizados procedimentos não invasivos neste dia, tais como radiografias: panorâmica e periapical na região dos incisivos inferiores e planejado a raspagem gengival supra e subgengival se necessário. Devido à hiperglicemia e dificuldade de controle da mesma, e, sabendo-se que a raspagem poderia ocasionar sangramento, foi prescrito antibioticoterapia profilática para a próxima consulta. O antibiótico prescrito, amoxicilina $1 \mathrm{~g}$ antes do procedimento, foi eleito após consultar a médica endocrinologista da criança. Além disso, a paciente foi motivada a procurar por uma nutricionista e se alimentar conforme o plano nutricional proposto que contém os alimentos ideais para sua dieta, e a usar sua insulina de rotina para o controle glicêmico. 
No terceiro encontro com a criança se observou o valor da glicemia casual pré-consulta foi de $132 \mathrm{mg} / \mathrm{dL}$. Além da participação do pai que a acompanhava, havia realizado o uso da antibioticoterapia profilática como orientada e seguido a dieta da nutricionista. A criança foi elogiada devido a sua motivação em manter a glicemia controlada, o que proporcionou o tratamento odontológico. Foi possível fazer revelação do biofilme dental, instrução de higiene bucal, escovação supervisionada, raspagem supragengival nos incisivos inferiores em todas as faces, profilaxia com pasta profilática e escova tipo Robinson e aplicação tópica de flúor. Após a raspagem pôde-se confirmar o valor da glicemia casual pós-consulta, de $112 \mathrm{mg} / \mathrm{dL}$.

Costa et al. (2016) observa que não é necessário o uso de antibióticos nos procedimentos odontológicos de rotina em pacientes com diabetes, somente na presença de infecções bucais estes deveriam ser prescritos devido à baixa resistência e ao retardo da cicatrização. A antibioticoterapia profilática é indicada em pacientes descompensados de alto risco frente a procedimentos cirúrgicos invasivos imprescindíveis, presença de infecções bucais, atendimentos de urgência nos quais não seja possível determinar a classificação exata de risco apresentado pelo paciente. Pessoas com diabetes não controlado apresentam maior susceptibilidade a infecções crônicas e inflamações dos tecidos orais.

Fernandes et al. (2015) em seu estudo sobre cicatrização de dentes extraídos observaram que se pode inferir que pessoas com diabetes tipo 2 submetidas a extrações dentárias de dentes que não apresentam infecção odontogênica aguda não devem receber profilaxia antibiótica simplesmente por causa de seu status diabético ou nível de controle glicêmico.

A criança participante deste projeto de extensão não tinha nenhuma infecção bucal, no entanto, a decisão sobre a antibioticoterapia profilática foi baseada no histórico do valor da hemoglobina glicada da paciente, que vinha se apresentando com valores acima dos desejáveis há algum tempo segundo relato da médica quando consultada sobre a antibioticoterapia profilática. Também a certeza do sangramento que o procedimento causaria foi um fator que incidiu para essa decisão. Além disso, foi importante a orientação nutricional realizada pela nutricionista para que hábitos saudáveis e motivação para uma boa dieta alimentar fossem incorporados na rotina da paciente. A boa glicemia permitiu com que os profissionais de Odontologia, realizassem o procedimento com segurança.

No quarto encontro, a glicemia casual pré-consulta da paciente estava $53 \mathrm{mg} / \mathrm{dL}$, caracterizando um quadro de hipoglicemia, apesar da paciente não apresentar nenhuma sintomatologia. Um sachê de mel foi oferecido para a paciente e minutos depois a glicemia estava $91 \mathrm{mg} / \mathrm{dL}$. A hiperplasia gengival na região do rebordo alveolar ântero-inferior estava menos acentuada, gengiva pouco sangrante e havia pouca quantidade de cálculo dental o que 
demonstrou uma melhora significante do quadro de gengivite. Foi realizada uma nova instrução de higiene bucal, escovação supervisionada, profilaxia com pasta profilática e aplicação tópica de flúor.

A gengivite regrediu no intervalo de uma semana, após a primeira sessão de raspagem supragengival. Porém, foram necessárias quatro sessões subsequentes para obter um periodonto saudável e motivação da paciente. Na última sessão foi possível realizar a restauração classe IV em resina composta do dente 21.

A incidência do DM tem aumentado progressivamente. Cerca de 50\% das pessoas com DM desconhecem o seu diagnóstico (FIALHO et al., 2012). Reconhecer os sintomas, a fim de realizar um diagnóstico precoce da doença, nos casos em que o paciente não tem ciência da doença, ou de tratá-la adequadamente quando o paciente já está diagnosticado com a moléstia, é fundamental.

A avaliação do estado geral de saúde e a adoção de medidas preventivas aumentam a segurança clínica no atendimento de pacientes que requerem cuidados especiais (SANTOS e RUMEL et al., 2006). Por isso, inicialmente a anamnese criteriosa foi estabelecida, nela a história médica, odontológica e relações familiares foram os principais aspectos abordados.

A avaliação da história médica é de grande importância no tratamento desses pacientes, pois por meio dela é possível realizar contato com o médico da criança frente a uma emergência ou dúvida, além da investigação das medicações utilizadas. No caso relatado, a avó da paciente mencionou que a moléstia havia sido descoberta em seu organismo em 2009, quando necessitou de uma internação na UTI devido a uma cetoacidose. Lalla e D'ambrosio, (2001) relataram que a resistência à insulina no DM resulta na inabilidade das células dependentes de insulina de fazerem uso da glicose. Além disso, triglicerídeos armazenados são quebrados em ácidos graxos como uma alternativa de combustível e há uma elevação no nível de corpos cetônicos no sangue, levando o paciente a cetoacidose. A paciente utiliza insulina NPH e regular como medicação. Durante o período do tratamento odontológico ela sofreu diversos quadros de hiperglicemia. A avó tinha dificuldade de monitorar a dieta da paciente, além disso, a alimentação não era balanceada e nem acompanhada por nutricionista. Devido aos episódios de hiperglicemia, uma dieta balanceada foi fundamental para o controle metabólico da paciente, por isso o atendimento multiprofissional.

A história odontológica demonstrou que a paciente nunca havia sido submetida a uma anestesia odontológica. Fernandes et al., (2010) e Neto et al. (2012) refletem em seus estudos sobre o cuidado ao utilizar soluções anestésicas contendo adrenalina, uma vez que o hormônio provoca a quebra do glicogênio em glicose, podendo ocasionar hiperglicemia, realizando o efeito 
contrário ao da insulina. No entanto, Costa et al. (2016) ressalvam que nos pacientes compensados o uso de anestésico com vasoconstrictor adrenérgico é permitido, diferentemente de pacientes descompensados e com possível presença de comorbidades, quando seria mais sensato o anestésico prilocaína com o vasoconstrictor felipressina, por ser tratar de um vasoconstrictor hormonal de pequeno impacto sobre a condição cardiovascular.

Sales et al. (2009) relatou que há dificuldade de a criança com diabetes aceitar sua condição existencial, pois sente-se diferente das demais crianças. Além disso, toda a rotina familiar é alterada, criando um sentimento de inferioridade da própria criança. Investigando a relação familiar, pôde-se observar que a criança teve uma melhora significativa na autoestima quando acompanhada pelo pai, mesmo não sendo a pessoa com quem convive diariamente. A adesão ao tratamento, cuidados com a higiene bucal e até mesmo em relação à própria dieta, tiveram uma resposta positiva quando o convívio com o pai aumentou nesta fase. A investigação das experiências vividas pela criança, tanto na escola, como no ambiente familiar, permitiu que a equipe multiprofissional melhorasse o envolvimento da criança. Concordando com o que afirmou Sparapani et al. (2009), o aumento do envolvimento dos pais com os jovens no manejo do diabetes, encorajados por uma equipe de educação em saúde, pode resultar no crescimento do envolvimento de ambos no tratamento e consequente melhora dos resultados glicêmicos.

Durante o exame físico intrabucal, pôde-se observar a presença de cálculo supragengival nos incisivos inferiores, nas faces vestibular e lingual. Apresentava também hiperplasia gengival e gengivite no rebordo alveolar ântero-inferior. Os tecidos periodontais são as estruturas mais acometidos pelo DM. Vieira e Recchioni, (2008) relatou que crianças DMT1, com baixo controle metabólico, geralmente iniciam o processo de periodontite em torno da puberdade. Sabendo que a falta de controle do cálculo pode induzir a periodontite avançada, o planejamento primeiramente adotado foi em função da remoção desta.

O controle glicêmico no encontro inicial, assim como nos subsequentes, foi fundamental para guiar as decisões clínicas. Segundo a American Diabetes Association, o controle glicêmico é fundamental para condução do diabetes, no qual o índice de glicose sanguínea está associado à diminuição do padrão de muitas complicações sistêmicas (AMERICAN DIABETES ASSOCIATION, 2019). Somente por meio da aferição glicêmica casual foi possível identificar o quadro de hipoglicemia no quarto encontro, e de hiperglicemia no primeiro e segundo encontros, pois em nenhum deles os quadros foram sintomáticos.

$\mathrm{O}$ atendimento interprofissional e multiprofissional mostrou-se eficaz principalmente no terceiro encontro, quando se conseguiu realizar o atendimento da paciente sem complicações, pois foi possível o controle da glicemia, com a orientação e motivação realizada pela nutricionista. 
A aferição e as instruções dadas aos familiares e à criança para o controle glicêmico possibilitaram a realização dos procedimentos, além de terem sido imprescindíveis os contatos realizados com a médica endocrinologista.

Durante o quadro de hipoglicemia, ocorrido no quarto encontro, apesar de ser assintomático, decidiu-se em oferecer um sachê de mel à paciente, para que o atendimento fosse realizado com tranquilidade. Segundo Alves et al., (2006) para o tratamento do quadro de hipoglicemia, o paciente consciente deverá ingerir o equivalente a 15 gramas de glicose: 1 comprimido de glicose ou 1 sachê de mel, por exemplo. Nery, (2008) relatou que a ocorrência de hipoglicemia sem sinais de alarme foi descrita há mais de 50 anos em pessoas com DMT1, isso ocorre porque há pacientes que toleram concentrações glicêmicas muito baixas sem os sintomas de alarme que permitem que o paciente reconheça o episódio e tome as providências adequadas para abortá-lo. Os sinais e sintomas são adrenérgicos (tremor, sudorese, palidez, taquicardia, palpitação e fome) e neuroglicopênicos (cefaleia, tontura, sonolência, irritabilidade, visão turva, confusão mental, desmaio, convulsão e coma) (KAUR et al., 2009). A manutenção da consulta foi decidida com o embasamento da literatura que relata a elevação da glicemia de um paciente com hipoglicemia utilizando um sachê de mel, por meio da experiência pessoal da paciente nessas situações e também com a realização de um outro teste para verificação da glicemia após 25 minutos da ingestão do sachê.

O reforço, a cada encontro, de orientação de higiene bucal, possibilitou uma melhora progressiva, obtendo um periodonto mais saudável.

Com o conhecimento sobre as manifestações e complicações do DMT1 foi possível realizar um atendimento criterioso, resultando no sucesso da conclusão do caso clínico.

\section{CONSIDERAÇÕES FINAIS}

Pôde-se observar a importância de uma equipe interprofissional que o projeto de extensão proporciona, assim como o contato com o médico e nutricionista que acompanham a criança, para que o interprofissionalismo e o multiprofissionalismo ampliassem o olhar dos profissionais em relação ao estado de saúde geral da paciente. Considera-se que a atuação interprofissional e multiprofissional no planejamento e no desenvolvimento das atividades, favoreceram a interlocução de conhecimentos e experiências para ajudar na resolução das situações vivenciadas, promovendo, assim, melhores condições para o paciente lidar com sua saúde geral e bucal. 


\section{REFERÊNCIAS}

AGUIAR, D. G. et al. Atendimento Odontológico ao paciente diabético tipo 1. Odontologia Clín. Científi., Recife, v. 8, n. 1, p. 13-19, jan./mar. 2009.

ALVES, C. et al. Atendimento odontológico do paciente com diabetes melito: recomendações para a prática clínica. Rev. Ci. Med. Biol., Salvador, v. 5, n. 2, p. 97-110, maio/ago. 2006. ALVES, C. et al. Mecanismos patogênicos da doença periodontal associada ao diabetes mellitus. Arq. Bras. Endocrinol. Metab. São Paulo, v. 51, n.7, p. 1050-1057, out. 2007.

AMERICAN DIABETES ASSOCIATION. Classification and Diagnosis of Diabetes: Standards of Medical Care in Diabetes-2019. American Diabetes Association. Diabetes Care Jan 2019, 42 (Supplement 1) S13-S28. [acesso em 2020 abr. 01]. Disponível em: https://care.diabetesjournals.org/content/42/Supplement_1/S13

ANGIONI M.; VICENTINI M. A. Percepções de estudantes egressos de projetos de extensão sobre a extensão universitária da FURB. Participação revista de extensão da Universidade de Brasília - Conhecimento aplicado na prática. Brasília. nº 27, 2015.

ASSOCIAÇÃO NACIONAL DE ATENÇÃO AO DIABETES (ANAD). [acesso em 2020 abr. 01]. Disponível em: https://www.anad.org.br/o-que-deve-saber-sobre-diabetes/

BORGES, A. et al. Tratamento endodôntico de pacientes diabéticos: um relato de caso clínico. Perspect Oral Sci. v.2, n.2, p.37-42, ago. 2010.

BOTERO, J. E. et al. Effects of periodontal non-surgical therapy plus azithromycin on glycemic control in patients with diabetes: a randomized clinical trial. Antioquia: J Period. Res. v.48, n.6, p.706-712, dec. 2013.

CIRIACO, E.V.; BUENDÍA, M. C. L. Multi-disciplinary treatment of female patient afflicted with generalized aggressive periodontitis and type 1 diabetes mellitus. Revista Odontológica Mexicana. v.18, n.1, p.32-37, jan./mar. 2014.

COSTA, R. M; TEIXEIRA, L. G; AZOUBEL, E; AZOUBEL, M. C. F; AZEVEDO, F. C. G. Pacientes diabéticos na clínica odontológica: diretrizes para o acolhimento e atendimento.

Brasileira de Ciências da Saúde. V. 20, n. 4), p. 333-340, 2016

FEDERAÇÃO INTERNACIONAL DE DIABETES. Atlas de Diabetes da IDF, $9 a$ edn. Bruxelas, Bélgica: 2019. Disponível em: https://www.diabetesatlas.org

FERNANDES, P. M., et al. Abordagem odontológica em pacientes com diabetes mellitus tipo 1. Pediatria. São Paulo, v.32, n.4, p. 274-80, 2010.

FERNANDES, K. S.; GLICK, M ; SOUZA, MS ; KOKRON, CM ; GALLOTTINI, M. H. C. . Association between immunologic parameters, glycemic control and post-extraction complications in individuals with type 2 diabetes. The Journal of the American Dental Association, v. 146, p. 592-599, 2015.

FIALHO, P.G.V.; ARAUJO, M.A.R.; ARAUJO, P.H.P.A. Cuidados no atendimento odontológico do paciente portador de diabetes mellitus. Rev Cienc Saude. São Luís, v.14, n.2, p.103-112, 2012. 
A participação da extensão na resolução de caso de criança com Diabetes Mellitus Tipo 1

KAUR, G. Association betweentype 1 and type 2 diabetes with periodontal disease and too thloss. J. clin. Periodontol. v.36, n.9, p.765-774, 2009.

LALLA, R.V.; D'AMBROSIO, J.A. Dental management considerations for the patient with diabetes mellitus. The Journal of the American Dental Association. v.132, n.10, p.1425-1432, 2001.

LIMA L.O; LIMA G.O; ALVES F.S; CARATIN, W. W. C.; FERNANDES, K. S.; TOBOUTI, P. L. . Diabetes e Odontologia. Journal of Biodentistry and Biomaterials, v. 5, p. 6-13, 2015.

LOPES DV, OLIVEIRA F, BATISTA GS, BOTELHO MC, SANTOS NA, SILVA RBV, ET AL. Adesão ao tratamento para pacientes com diabetes mellitus tipo $2 \mathrm{em}$ unidades básicas de saúde do município de Alfenas-MG. J Health Sci Inst, v. 37, n.2, p. 123-8, 2019.

NERY, M. Hipoglicemia como fator complicador no tratamento do diabetes mellitus tipo 1. Arq Bras Odontol. São Paulo, v.52, n.2, p.288-298, mar. 2008.

NETO, J.N.C., et al. O paciente diabético e suas complicações para conduta odontológica. Rev. Dent. onl. Feira de Santana, v. 11, n.23, 2012.

OLIVEIRA, MF. DAMO, N.G., RAITZ, I.W., VEIGA, M.L., PEREIRA, L. Cuidados odontológicos em pacientes diabéticos. Arq. Catarin Med, v. 48, n.3 jul.-set., p. 158-170, 2019.

SALES, C.A. et. al. O cuidar de uma criança com diabetes mellitus tipo 1: concepções dos cuidadores informais. Rev. Eletrônica Enferm. Maringá, v.11, n.3, p.563-72, 2009.

SANTOS, P. S.; JUNIOR, L. A. V.S. Medicina bucal: a prática da odontologia hospitalar. São Paulo, Santos.2009.

SANTOS, J.C.; RUMEL, D. Emergência médica na prática odontológica no Estado de Santa Catarina: ocorrência, equipamentos e drogas, conhecimento e treinamento dos cirurgiõesdentistas. Ciênc. Saúde Colet. Tubarão, v.11, n.1, p.183-190, 2006.

SANTOS, M.F.D. et al. Abordagem odontológica do paciente diabético um estudo de intervenção. Odontol clín-cient. Recife, v.9, n.4, p.319-324, dez., 2010.

SOCIEDADE BRASILEIRA DE DIABETES (SBD). DIRETRIZES Sociedade Brasileira de Diabetes 2019-2020. São Paulo, p, 491, 2020. [acesso em 2020 abr. 01]. Disponível em: https://www.diabetes.org.br/profissionais/images/DIRETRIZES-COMPLETA-2019-2020.pdf

SPARAPANI, V.C.; NASCIMENTO, L.C. Crianças com diabetes mellitus tipo 1: fortalezas e fragilidades no manejo da doença. Ciênc. Cuid. Saúde. Ribeirão Preto, v.8, n.2, p. 274-279,2009.

TERRA, B.G.; GOULART, R.R.; BAVARESCO, C.S. O cuidado odontológico do paciente portador de diabetes mellitus tipo 1 e 2 na atenção primária a saúde. Rev. APS, Rio Grande do Sul, v.14, n.2, p.149-161, 2011.

VIEIRA, T.R. et al. Relação entre periodontite e diabetes mellitus em crianças e adolescentes. Arq Bras Odontol. Minas Gerais, v.4, n.2, p. 92-95, 2008. 
A participação da extensão na resolução de caso de criança com Diabetes Mellitus Tipo 1

WITT, A.R.S. et al. Marcadores Imunológicos da Diabetes Mellitus do tipo 1 - Revisão. Rev.

Conhecim. onl. Novo Hamburgo, v.2, n.3, set., 2012.

WORLD HEALTH ORGANIZATION (WHO). Definition, Diagnosis and Classification of Diabetes Mellitus and its Complications. Report of a WHO Consultation. Part 1: Diagnosis and Classification of Diabetes Mellitus. 1999.

Recebido em: 25/06/2019

Aceito em: 13/04/2020 